\title{
QUEUEING ANALYSIS OF MOBILE ADHOC NETWORKS EQUIPPED WITH COGNITIVE RADIO TECHNOLOGY
}

\author{
Neeta Singh and Jyoti Singh \\ School of Information and Communication Technology, Gautam Buddha University, India
}

\begin{abstract}
In this paper, the Mobile Ad Hoc Networks (MANETs) with integrated Primary/Secondary User (PU or SU) traffic has been considered. Nodes in the MANET are equipped with Cognitive Radio Technology (CRT). The blocking probability and dropping probability for $S U$ s are obtained by considering a 3D Markov Chain analytical model. To provide efficient service to PUs, we offer priority of channels, higher priority and lower priority is given to PUs and SUs, respectively. The expressions for blocking probability, dropping probability, throughput and non-completion of $S U$ are established. Sensitivity analysis is carried out to explore the effect of system parameters. It is observed that Ad Hoc network utilized the unused spectrum of the existing legacy systems in an opportunistic manner in addition to using the unlicensed spectrum. The results show an improved performance in terms of blocking probabilities, dropping probabilities of $S U$ and over all throughput of the system.
\end{abstract}

\section{Keywords:}

Mobile Ad Hoc Network, Cognitive Radio Technology, Primary Users, Secondary Users, Blocking Probability, Dropping Probability

\section{INTRODUCTION}

Owing to the scarcity of the frequency spectrum, cognitive radio technology (CRT) has grown tremendously, as the frequency channels can be used many times to facilitate efficient utilization of limited bandwidth available for MANETs. As radio spectrum is a scarce resource, the basic dimensioning issue in MANETs is to utilize the unlicensed, so that the prescribed level of Quality of service (QoS) is achieved. CRT is an intelligent technology which enables better utilization of the scarce radio frequency spectrum. The main objective of CRT is to detect and share unused spectrum without any interference. Spectrum sensing is the first building block of a CRT. The CRT is used to find the presence of PU in the network ([1], [2]). In case of PU is absent, CRT allows SU opportunistically to use the spectrum subject to the condition that it does not cause interference to the PU [3].

The PU can access the spectrum at any time. Whereas the SU can use the unused spectrum in a time basis without disturbing the PUs. The SU can use both the licensed and unlicensed band. Zhu et al. [3] analyzed a spectrum in the form of Markov chain model and also derived its blocking and dropping probabilities for the SU operating only in licensed band. The behavior of open spectrum accessed in unlicensed band is determined by author in an article [5]. A fair MAC protocol has been proposed in [6] - [9] with QOS provisioning for a MANETs. However, its analytical model has not been investigated.

In this paper, we have considered MANETs with integrated PU/SU traffic. The model description along with underlying assumptions and notations are given in section 2. A 3D markov model with the expressions for blocking probability $\left(P_{\text {blocking }}\right)$, dropping probability $\left(P_{\text {dropping }}\right)$ and throughput are computed in section 3 . The procedure to compute $P_{\text {blocking }}$ and $P_{\text {dropping }}$ and other performance parameters are also described. Numerical illustrations to validate the analytical results are facilitated in section 4. Finally, the contribution of the work is highlighted in section 5 .

\section{ASSUMPTIONS AND NOTATIONS}

In this section, we have considered a three states Markov chain model to analyze the MANETs equipped with CRT. The assumptions and notations used in defining the model are as follows:

There are two types of spectrum pools i.e. (i) Licensed spectrum pool $\left(C_{1}\right)$ for PUs and (ii) Unlicensed spectrum pool $\left(C_{2}\right)$ for SUs.

- Higher priority and lower priority is given to PUs and SUs, respectively.

- There is an equal probability of using unused channel by both the user.

- Total number of channel in the system $T C=C_{1}+C_{2}$

- Let $\mathrm{u}$ be the number of PUs at any time $t$

- Total number of available channels for SUs will be $C_{1}+C_{2-}$ $u$

- There are three states for PUs and SUs

$\circ u=C_{1}$ are used by PU with arrival rate $\lambda_{1}$

$\circ v=\left(C_{1}-u\right)$ are used by SU with arrival rate $\lambda_{2}$

○ $w=\left(C_{2}\right)$ are used by SU with arrival rate of $\lambda_{3}$

- Service rate for above three conditions are $\mu_{1}, \mu_{2}$ and $\mu_{3}$.

- $\left(C_{1}-u\right)$ and $C_{2}$ are the two types of channels that are used by SUs.

- In the arrival of PUs, the SU vacate $C_{1}$ channel for PU and shift its services to another available $C_{1}$ or $C_{2}$.

。 The switching delay is less for SUs when it uses the $C_{1}$

○ The switching delay is more for SUs when it switches to the $C_{2}$

- Therefore, we will give more importance in $C_{1}$ spectrum to avoid switching delay

- The blocking probability of SU denoted by $P_{\text {blocking }}$

- The dropping probability of SU denoted by $P_{\text {dropping }}$

\section{THE ANALYTICAL MODEL}

In this section, we describe the analytical model to evaluate the performance of SU. Suppose $G_{1}(t), G_{2}(t)$ and $G_{3}(\mathrm{t})$ are the 
three states $(u, v, w)$ at a time $t$. Thus the three dimensional Markov process for $G_{1}(t), G_{2}(t), G_{3}(t)$ with state spaces $S$ is:

$$
S=\left\{(u, v, w) \mid 0 \leq u \leq C_{1}, 0 \leq v \leq C_{1}-u, 0 \leq w \leq C_{2}\right\} .
$$

As there are three states in the system from $(0,1,2)$. Initially we have considered $C_{1}=2$ and $C_{2}=2$. So at any time there may be maximum 4 users in the system. The Table.1 shows the total 18 states at $t$ time in this system.

Table.1. Number of states at a time $(t)$

\begin{tabular}{|c|c|c|c|c|}
\hline $\boldsymbol{u}, \boldsymbol{v}, \boldsymbol{w}$ & $\boldsymbol{u}$ & $\boldsymbol{v}$ & $\boldsymbol{w}$ & $\begin{array}{c}\text { Total no. of users } \\
\text { at time } \boldsymbol{t}\end{array}$ \\
\hline $0,0,0$ & No & No & No & 0 \\
\hline $0,0,1$ & No & No & Yes & 1 \\
\hline $0,1,0$ & No & Yes & No & 1 \\
\hline $1,0,0$ & No & No & No & 1 \\
\hline $2,0,0$ & Yes & No & No & 2 \\
\hline $1,0,1$ & Yes & No & Yes & 2 \\
\hline $0,0,2$ & No & No & Yes & 2 \\
\hline $1,1,0$ & Yes & Yes & No & 2 \\
\hline $0,1,1$ & No & Yes & Yes & 2 \\
\hline $0,2,0$ & No & Yes & No & 2 \\
\hline $2,0,1$ & Yes & No & Yes & 3 \\
\hline $1,0,2$ & Yes & No & Yes & 3 \\
\hline $1,1,1$ & Yes & Yes & Yes & 3 \\
\hline $0,1,2$ & No & Yes & Yes & 3 \\
\hline $0.2,1$ & No & Yes & Yes & 3 \\
\hline $2,0,2$ & Yes & No & Yes & 4 \\
\hline $1,1,2$ & Yes & Yes & Yes & 4 \\
\hline $0,2,2$ & No & Yes & Yes & 4 \\
\hline
\end{tabular}

The Chapman-Kolmogorov equations governing the model are as follows: we can also make state transition diagram with the help of theses equations which are as follows.

$$
\begin{aligned}
& \left(\lambda_{1}+\lambda_{2}\right)_{P(0,0,0)}=\mu_{1_{P(1,0,0)}}+\mu_{2_{P(0,0,1)}}+\mu_{2_{P(0,1,0)}} \\
& \left(\lambda_{1}+\lambda_{2}+\mu_{2}\right)_{P(0,0,1)}=\mu_{1_{P(1,0,1)}}+2 \mu_{2_{P(0,0,2)}}+\mu_{2_{P(0,1,1)}}+\frac{\lambda_{2_{P(0,0,0)}}}{2} \\
& \left(\lambda_{1}+\lambda_{2}+\mu_{2}\right)_{P(0,1,0)}=\mu_{1_{P(1,1,0)}}+\mu_{2_{P(0,1,1)}}+2 \mu_{2_{P(0,2,0)}}+\frac{\lambda_{2_{P(0,0,0)}}}{2} \\
& \left(\lambda_{1}+\lambda_{2}+\mu_{1}\right)_{P(1,0,0)}=2 \mu_{2_{P(2,0,0)}}+\mu_{2_{P(1,0,1)}}+\mu_{2_{P(1,1,0)}}+\lambda_{1_{P(0,0,0)}} \\
& \left(\lambda_{1}+\lambda_{2}+\mu_{1}+\mu_{2}\right)_{P(1,0,1)}=2 \mu_{1_{P(2,0,1)}}+2 \mu_{2_{P(1,0,2)}}+\mu_{2_{P(1,1,1)}} \\
& +\frac{\lambda}{3}_{P(0,1,0)}+\lambda_{1_{P(0,0,1)}}+\frac{\lambda}{2}_{P(1,0,0)} \\
& \left(\lambda_{1}+\lambda_{2}+2 \mu_{2}\right)_{P(0,0,2)}=\mu_{1_{P(1,0,2)}}+2 \mu_{2_{P(0,1,2)}}+\frac{\lambda}{2}_{P(0,0,1)} \\
& \left(\lambda_{1}+\lambda_{2}+\mu_{1}+\mu_{2}\right)_{P(1,1,0)}=\mu_{2_{P(1,1,1)}}+{\frac{2 \lambda_{2}}{3}}_{P(0,1,0)}+\frac{\lambda}{2}_{P(1,0,0)}
\end{aligned}
$$

$$
\begin{aligned}
& \left(\lambda_{1}+\lambda_{2}+3 \mu_{2}\right)_{P(0,1,1)}=\mu_{1_{P(1,1,1)}}+2 \mu_{2_{P(0,1,2)}}+2 \mu_{2_{P(0,2,1)}} \\
& +\frac{\lambda}{2}_{P(0,1,0)}+\frac{\lambda}{2}_{P(0,0,1)} \\
& \left(\lambda_{1}+\lambda_{2}+2 \mu_{2}\right)_{P(0,2,0)}=\mu_{2_{P(0,2,1)}}+{\frac{\lambda_{2}}{2}}_{P(0,1,0)} \\
& \left(\lambda_{2}+2 \mu_{1}\right)_{P(2,0,0)}=\lambda_{1_{P(1,0,0)}}+\mu_{2_{P(2,0,1)}} \\
& \left(\lambda_{1}+\lambda_{2}+\mu_{2}+\mu_{1}\right)_{P(1,1,1)}=\lambda_{2_{P(1,1,0)}}+\frac{2 \lambda_{1}}{3}{ }_{P(0,1,1)}+\lambda_{1_{P(0,2,0)}} \\
& +\frac{\lambda}{2}_{P(1,0,1)}+2 \mu_{2_{P(1,1,2)}} \\
& \left(\lambda_{1}+\lambda_{2}+3 \mu_{2}\right)_{P(0,2,1)}=2 \mu_{2_{P(0,2,2)}}+\frac{\lambda}{2}_{P(0,1,1)}+\lambda_{2_{P(0.2,0)}} \\
& \left(\frac{5 \lambda_{1}}{3}+\lambda_{2}+2 \mu_{2}\right)_{P(0,1,2)}=2 \mu_{2_{P(0,2,2)}}+\mu_{1_{P(1,1,2)}}+\lambda_{2_{P(0,0,2)}}+{\frac{\lambda_{2}}{2}}_{P(0,1,1)} \\
& \left(\lambda_{1}+\lambda_{2}+\mu_{1}+2 \mu_{2}\right)_{P(1,0,2)}=2 \mu_{1_{P(2,0,2)}}+\mu_{2_{P(1,1,2)}}+\frac{\lambda_{1}}{3}{ }_{P(0,1,2)} \\
& +\frac{\lambda}{3}_{P(0,1,1)}+\lambda_{1_{P(0,0,2)}}+\frac{\lambda_{1}}{2} \\
& \left(\lambda_{2}+2 \mu_{1}+\mu_{2}\right)_{P(2,0,1)}=2 \mu_{2_{P(2,0,2)}}+\lambda_{1_{P(1,1,0)}}+\lambda_{1_{P(1,0,1)}}+\lambda_{1_{P(2,0,0)}} \\
& \left(2 \mu_{1}+2 \mu_{2}\right)_{P(2,0,2)}=\lambda_{1_{P(1,1,2)}}+\lambda_{1_{P(1,1,1)}}+\lambda_{1_{P(1,0,2)}}+\lambda_{2_{P(2,0,1)}} \\
& \left(\lambda_{1}+\mu_{1}+3 \mu_{2}\right)_{P(1,1,2)}=\lambda_{1_{P(0,2,2)}}+\lambda_{1_{P(0,2,1)}}+\frac{\lambda_{1}}{3} \\
& +\lambda_{2_{P(1,1,1)}}+\lambda_{2_{P(1,0,2)}} \\
& \left(\lambda_{1}+4 \mu_{2}\right)_{P(0,2,2)}=\lambda_{2_{P(0,1,2)}}+\lambda_{2_{P(0,2,1)}}
\end{aligned}
$$

The equilibrium probability distribution of $G_{1}(t), G_{2}(t)$ and $G_{3}(t)$ states are assumed to be $P_{1 u}, P_{2 v \mid u}$ and $P_{3 w}$. $G_{2}(t)$ is $P_{2 v \mid u}$ which shows that PUs already present in the system. Thus, the joint probability distribution of $p_{(u, v, w)}$ can be written as

$$
\begin{gathered}
P_{(u, v, w)}=P_{1_{u}} P_{2_{v \mid u}} P_{3_{w}} \\
P_{0}=P_{1_{0}} P_{2_{0 \mu}} P_{3_{0}}
\end{gathered}
$$

When SUs gets alarm by PUs, the equilibrium conditional probability distribution $P_{1 u}$ can be achieved. Thus the SUs can be modeled as $M / M / C_{1}-u$, queuing system for $u=0,1,2, \ldots, C_{1}$. Therefore by considering $M / M / C_{1}-u$ queuing system, we can calculate the conditional probability distribution $P_{2 v \mid u}$ [7].

$$
P_{1_{u}}=\frac{1}{u !} \rho_{1}^{u} \rho_{1_{0}} \quad 0 \leq v \leq C_{1}-u
$$

where, $\rho_{1}=\frac{\lambda_{1}}{\mu_{1}}$.

Steady state probability distribution of $\mathrm{P}_{2 v \mid u}$ for ongoing PUs is stated below. The ongoing PUs will not get affected by SUs. Hence, the presence of SUs is considered to be negligible. Therefore, the number of PUs in progress can be derived by $M / M / C_{1}$ queuing system. The probability distribution $P_{2 v \mid u}$ can be 
derived by using the standard $M / M / C_{1}$ queuing system formula from [7].

$$
P_{2_{v \mid u}}=\frac{1}{v !} \rho_{2}^{v} \rho_{2_{0} \mid v} \quad 0 \leq v \leq C_{1}-u
$$

where, $\rho_{2}=\frac{\lambda_{2}}{\mu_{2}}$.

By using standard $M / M / C_{2}$ queuing formula, the probability distribution of $P_{3 w}$ can be obtained. The SUs are not affected by any users in the unlicensed channels. Therefore,

$$
P_{3_{w}}=\frac{1}{w !} \rho_{3}^{w} \rho_{3_{0}} \quad 0 \leq w \leq C_{2}
$$

where, $\rho_{3}=\frac{\lambda_{3}}{\mu_{3}}$.

When both the licensed channels $\left(C_{1}\right)$ and unlicensed channels $\left(C_{2}\right)$ are occupied by PUs, SUs. The SUs services get blocked.

$$
p_{u, v, w}=\frac{\rho_{1}^{u} \rho_{2}^{v} \rho_{3}^{w}}{u !, v !, w !} p_{0} 0 \leq u \leq C_{1}, 0 \leq v \leq C_{1-u} 0 \leq w \leq C_{2}
$$

From normalized state, sum of all the probability is equal to 1 .

$$
\sum_{u=0}^{C_{1}} \sum_{v=0}^{C_{1}-u} \sum_{k=0}^{C_{2}} p_{u, v, w}=1
$$

By replacing the probability, $P_{u, v w}$ from Eq.(24) to Eq.(25), we can solve the equation to calculate $P_{0}$,

$$
\begin{gathered}
\sum_{u=0}^{C_{1}} \sum_{v=0}^{C_{1}-u} \sum_{k=0}^{C_{2}} \frac{\rho_{1}^{u} \rho_{2}^{v} \rho_{3}^{w}}{u !, v !, w !} p_{0}=1 \\
p_{0}=\left[\sum_{u=0}^{C_{1}} \frac{\rho_{1}^{u}}{u !} \sum_{v=0}^{C_{1}-u} \frac{\rho_{2}^{v}}{v !} \sum_{k=0}^{C_{2}} \frac{\rho_{3}^{w}}{w !}\right]^{-1}
\end{gathered}
$$

\subsection{PERFORMANCE MEASURES}

Once the probabilities are computed, the performance measures are easily obtained. Using probabilities obtained in previous section, we can establish various performance measures as follows:

- The blocking probability $\left(P_{\text {blocking }}\right)$ of the SU: When all the $C_{1}-u$ channels are busy, the $\mathrm{SU}$ will be blocked with probability $P_{\text {blocking. }}$.

$$
\begin{gathered}
p_{\text {blocking }}=\sum_{u=0}^{c_{1}} \lambda_{2} p_{u}, C_{1}-u, C_{2} \\
p_{\text {blocking }}=\sum_{u=0}^{c_{1}} \lambda_{2} \frac{\rho_{1}^{u} \rho_{2}^{v} \rho_{3}^{w}}{u ! C_{1}-u !, C_{2} !} p_{0}
\end{gathered}
$$

- $\mathrm{SU}$ will be dropped with probability $p_{\text {dropping }}$, if all the $C_{1}$ and $C_{2}$ are busy

$$
\begin{gathered}
p_{\text {dropping }}=\sum_{u=0}^{C_{1}-u} \frac{\lambda_{1}}{C_{1}-u} p_{u}, C_{1}-u, C_{2} \\
p_{\text {dropping }}=\sum_{u=0}^{C_{1}-u} \frac{\lambda_{1}}{C_{1}-u} \frac{\rho_{1}^{u} \rho_{2}^{v} \rho_{3}^{w}}{u ! C_{1}-u !, C_{2} !} p_{0}
\end{gathered}
$$

The throughput $(T)$ for the SU will be the average number of service completed by SUs.

$$
T=\sum_{u=0}^{c_{1}} \sum_{v=0}^{c_{1}-u} \sum_{w=1}^{c_{2}}(v+w)\left(1-p_{n c}\right) p_{u, v, w}
$$

Hence probability of non-completion of SU services is given by $P_{n c}$.

$$
P_{n c}=P_{\text {blocking }}+\left(1-P_{\text {blocking }}\right) P_{\text {dropping }}
$$

\section{NUMERICAL RESULTS}

In this section, we have presented numerical experiments to validate the analytical results presented in above section. We perform the numerical experiment by taking example. The coding for this purpose is done in matlab software. Initially we have considered $C_{1}=2$ and $C_{2}=2$. So at any time there may be maximum 4 users in the system.

The purpose of sensitivity analysis presented in this section is to see how other performance measures vary with the input parameters $\lambda$ and $\mu$. Let us fix the system parameters as: $C_{2}=3$. The performance evaluation of SUs in evaluated in terms of above performance parameters i.e. $P_{\text {blocking }}, P_{\text {dropping }}$ and Throughput and shown in Fig.1-Fig.3) by varying the number of licensed channel $\left(C_{1}\right), C_{2}=3$ for SU as the IEEE $802.11 \mathrm{~b}$ standard supports three non-overlapping channels. In first case we have assumed both PUs and SUs are present in the system.

In Fig.1(a)-Fig.1(b) blocking probability of SU and Dropping probability of SU is depicted by varying arrival of PUs i.e. $\left(\lambda_{1}\right)$. It is observed that the blocking probability of SUs increases with the increase in arrival rate of PU as shown in Fig.1(a). This is due to increase in traffic of PUs, SUs has a higher probability to be interrupted hence dropping probability also increases with the increase in PUs. In Fig.1(b) the dropping probability is high with the increase in arrival rate of the PUs, which is quite obvious.

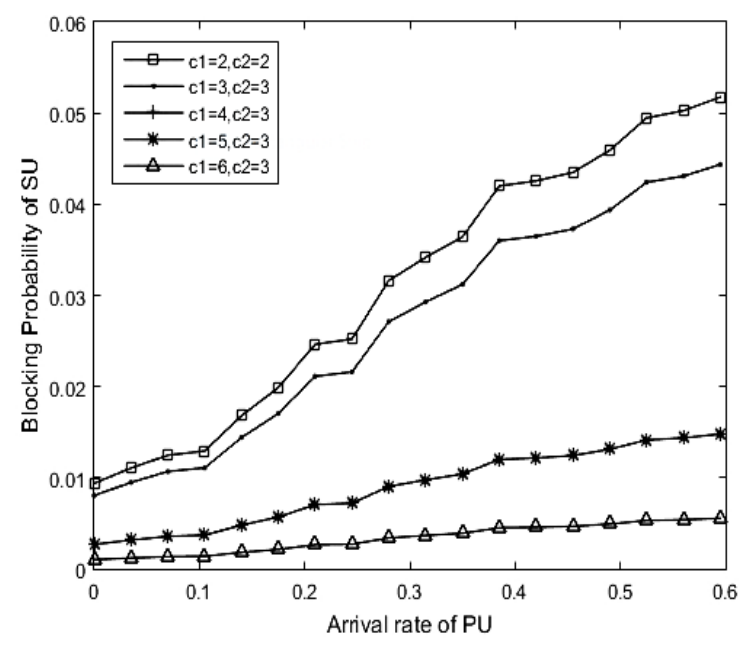

Fig.1(a). Effect of arrival rate $\left(\lambda_{1}\right)$ of PUs on blocking probability of SU 


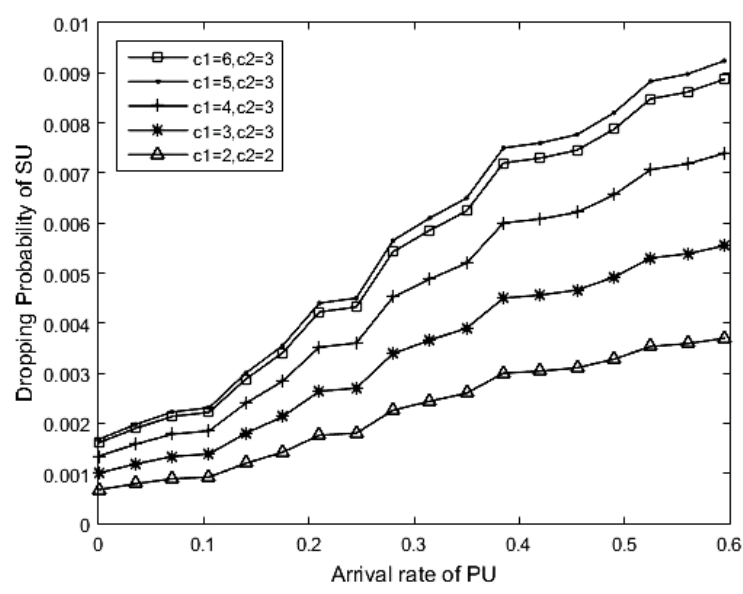

Fig.1(b). Effect of arrival rate $\left(\lambda_{1}\right)$ of PUs on Dropping probability of SU

The Fig.2(a)-Fig2(b) shows the effect of service rate PUs i.e. $(\mu 1)$ on blocking probability of SU and dropping probability of SU. In Fig.2(a) and Fig.2(b) it is also observed that, as the service rate increases the dropping probability of SU will still increase up to a maximum point and decrease when the holding time of PUs increases as we expected.

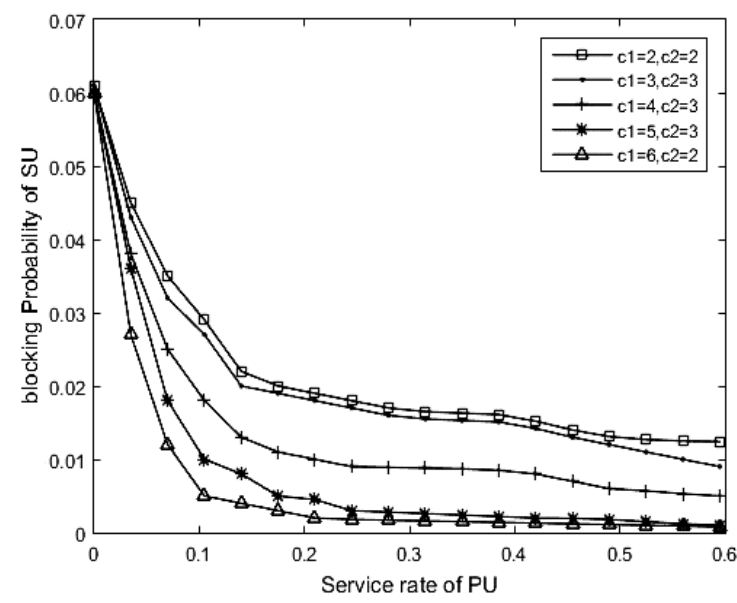

Fig.2(a). Effect of service rate $\left(\mu_{1}\right)$ of PUs on blocking probability of SU

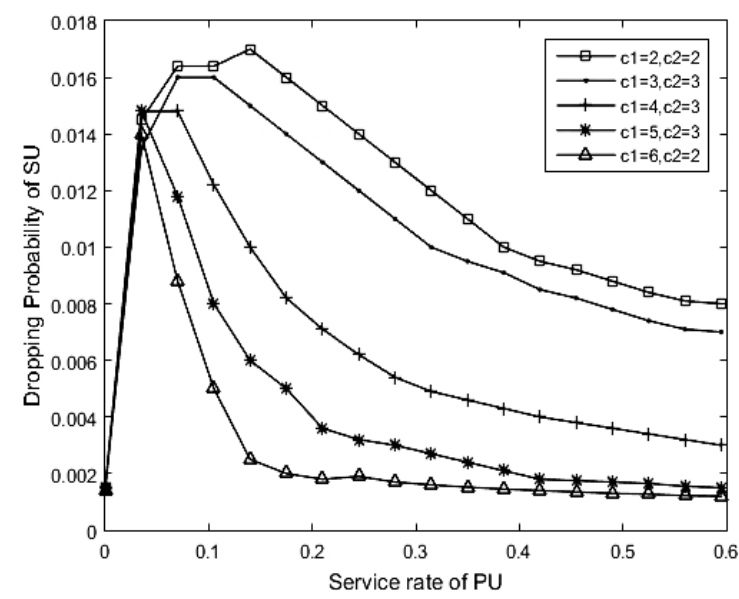

Fig.2(b). Effect of service rate $\left(\mu_{1}\right)$ PUs on dropping probability of $\mathrm{U}$
The Fig. 3 shows the Throughput of SUs vs. arrival rate of PUs. Under heterogeneous spectrum environment, the throughput of SUs when, $C_{1}=4,6$ is depicted in Fig.3. from the above illustrations, overall we conclude that with the help of CRT the throughput of the system is enhanced.

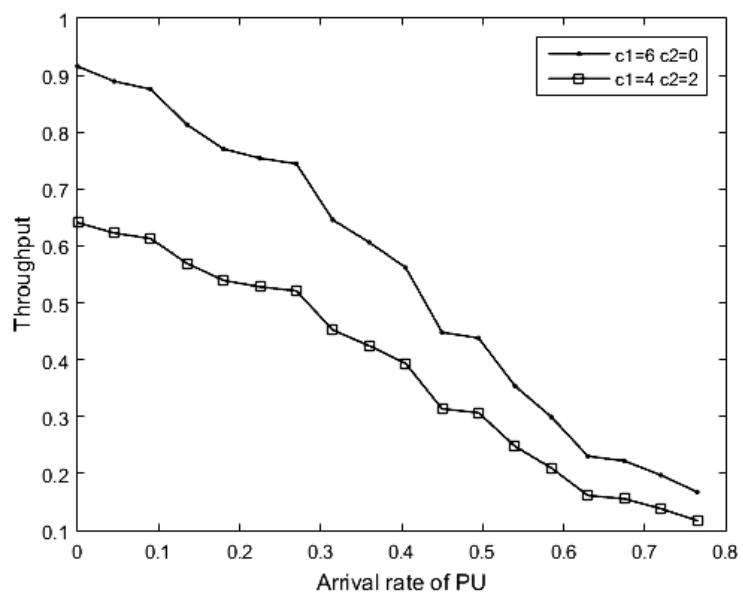

Fig.3. Throughput of SUs vs. arrival rate of PUs

\section{CONCLUSIONS}

We have studied the three-dimensional analytical model for MANETs equipped with CRT for sensing PUs and SUs. We have derived the expression for blocking probability of SUs, drooping probability of SUs, Throughput and probability of noncompletion of services of SUs. The analytical model developed is capable to deal with non-uniform pattern of traffic. Numerical experiments are performed which shows the validity of the analytical results with the effect of various parameters on system performance. The model may be helpful in providing guidelines for the design and performance prediction of CRT. Based on CRT the opportunistic procedure of assigning the channels can be easily implemented to support better QoS. The assumption of state-dependent arrival rate is taken into consideration which makes our model more feasible over a wide realistic range of situations occurring in licensed and unlicensed users. The computational tractability of the CRT is verified by taking numerical illustrations. We have facilitated sensitivity analysis which can be further used to determine the optimal control parameter.

\section{REFERENCES}

[1] Cisco, "Cisco Visual Networking Index: Forecast and Methodology, 2015-2020", Available at: https://www.cisco.com/c/en/us/solutions/collateral/serviceprovider/visual-networking-index-vni/white-paper-c11741490.html, Accessed on 2015.

[2] D. Farinacci, T. Li, S. Hnaks, D. Meyer, P. Traina and Juniper Networks, "Generic Routing Encapsulation (GRE)", Available at: https://tools.ietf.org/html/rfc2784.

[3] Peter Kietzmann, Cenk Gundogan, Thomas C. Schmidt, Oliver Hahm and Matthias Wählisch "The Need for a Name to MAC Address Mapping in NDN: towards Quantifying the Resource Gain", Proceedings of $4^{\text {th }}$ ACM Conference on Information-Centric Networking, pp. 1-6, 2017. 
[4] Satoshi Kodama, Rei Nakagawa, Toshimitsu Tanouchi and Shinya Kameyama, "Management System by using Embedded Packet for Hierarchical Local Area Network", Proceedings of IEEE International Conference on Ubiquitous Computing, Electronics and Mobile Communication, pp. 113-119, 2016.

[5] Satoshi Kodama, Rei Nakagawa and Toshimitsu Tanouchi, "Proposal of the Virtualized Control System for the Integrated Management of Multiple Services", Proceedings of IEEE $7^{\text {th }}$ International Conference and Workshop on Annual Computing and Communication, pp. 23-27, 2017.

[6] Satoshi Kodama, Rei Nakagawa and Toshimitsu Tanouchi, "A Research on the Integrated Virtual Platform for Managing Multiple Services", WSEAS Transactions on Information Science and Applications, Vol. 14, No. 12, pp. 102-111, 2017.
[7] Nick McKeown, Tom Anderson, Hari Balakrishnan, Guru Parulkar, Larry Peterson, Jennifer Rexford, Scott Shenker and Jonathan Turner, "OpenFlow: Enabling Innovation in Campus Networks", ACM SIGCOMM Computer Communication Review, Vol. 38, pp. 69-74, 2008.

[8] Rashid Mijumbi, Joan Serrat, Juan-Luis Gorricho, Niels Bouten, Filip De Turck and Raouf Boutaba, "Network Function Virtualization: State-of-the-Art and Research Challenges", IEEE Communications Surveys and Tutorials, Vol. 18, No. 1, pp. 236-262, 2018.

[9] J. Postel, "Transmission Control Protocol", Available at: https://tools.ietf.org/html/rfc793, Accessed on 1981.

[10] F. Gont and A. Yourtchenko, "On the Implementation of the TCP Urgent Mechanism", Available at: https://tools.ietf.org/html/rfc6093, 2011. 